Article

\title{
Multiple Silicon Nanowires with Enzymatic Modification for Measuring Glucose Concentration
}

\author{
Cheng-Chih Hsu ${ }^{1, *}$, Yu-Ching Liao ${ }^{1, \dagger}$, Yen-Ting Tsai ${ }^{1, \dagger}$, Hsin-I Yeh ${ }^{1, \dagger}$ and Chyan-Chyi Wu ${ }^{2, \dagger}$ \\ ${ }^{1}$ Department of Photonics Engineering, Yuan Ze University, 135, Yuan-Tung Road, Chung-Li 32003, \\ Taiwan; E-Mails: s1010757@mail.yzu.edu.tw (Y.L.); s1020721@ mail.yzu.edu.tw (Y.T.) \\ 2 Department of Mechanical and Electromechanical Engineering, Tamkang University, \\ New Taipei 25137, Taiwan; E-Mail: chyanchyi@gmail.com \\ $\dagger$ These authors contributed equally to this work. \\ * Author to whom correspondence should be addressed; E-Mail: cchsu@ saturn.yzu.edu.tw; \\ Tel.: +886-3-4638800 (ext. 7520); Fax: +886-3-451428.
}

Academic Editor: Joost Lötters

Received: 10 July 2015 / Accepted: 6 August 2015 / Published: 14 August 2015

\begin{abstract}
This study fabricated a multiple poly-Si nanowires sensor through a top-down method and immobilized glucose oxidase on the multiple nanowires for determining glucose concentration. The proposed sensor is $340 \mathrm{~nm}$ in width and uses five physically identical and parallel nanowires. The sensor contained nanowires of various lengths (3, 5, and $10 \mu \mathrm{m})$. Experimental results showed that sensor sensitivity is inversely proportional to nanowire length. The sensor with $3 \mu \mathrm{m}$ in nanowire length exhibited a theoretical resolution of $0.003 \mathrm{mg} / \mathrm{dL}$ and the highest sensitivity of $0.03 \mu \mathrm{A} /(\mathrm{mg} / \mathrm{dL})$. Furthermore, the proposed sensor retains this performance when reused for up to 10 applications.
\end{abstract}

Keywords: multiple nanowires; glucose sensor; enzymatic modification; top-down method

\section{Introduction}

In 2014, the U.S. Centers for Disease Control and Prevention (CDC) reported up-to-date scientific data on diabetes in the United States, revealing that 29.1 million (approximately 10\%) of the U.S. population has diabetes. In 2012, the estimated treatment cost for diabetes-related in the U.S. was approximately of U.S. $\$ 245$ billion. Many clinical studies have indicated that managing lower 
blood glucose levels can reduce risk factors for cardiovascular disease. Blood glucose levels can be efficiently self-monitored by using commercial glucose meters. According to the guideline suggested by the U.S. National Institute of Health (NIH), self-testing is often done before meals, after meals, and at bedtime for type 2 diabetes [1,2]. Therefore, to measure glucose concentration by using glucose meter is essential for reducing diabetes-related expenses. Several studies [3-7] have suggested the possibility of measuring glucose concentration by using nanowire-structure sensors. A nanowire sensor operates under the principle that the charge around the structure induces a surface potential at the nanowire surface, which is translated into a change in drain-to-source current $[8,9]$. Chen et al. [3] reviewed current literature regarding Si nanowire field effect transistors (FET) used for biosensing, and Rahman et al. [4] comprehensively reviewed nanostructured metal-oxide glucose biosensors. Rahman highlighted the advantages and disadvantages of enzymatic and nonenzymatic glucose sensors. Enzymatic glucose sensors exhibit high selectivity at an appropriate $\mathrm{pH}$ condition. Although nonenzymatic sensors have better $\mathrm{pH}$ immunity, less selectivity and quick inactivity of such sensors are still imperative improvements.

This study proposed a reusable enzymatic glucose sensor that fabricated a multiple poly-Si nanowires sensor by using a top-down method [3,10] and an immobilized glucose oxidase technique [11] on the nanowires to determine glucose concentration. The sensor has five physically identical parallel nanowires and the width of each nanowire is $340 \mathrm{~nm}$. The sensor contained nanowires of three lengths that controlled at 3, 5, and $10 \mu \mathrm{m}$. The experimental results revealed that sensitivity is inversely proportional to nanowire length; the highest sensitivity obtained was $0.03 \mu \mathrm{A} /(\mathrm{mg} / \mathrm{dL})$, the theoretical resolution of $0.003 \mathrm{mg} / \mathrm{dL}$, and the practical resolution was $1.23 \mathrm{mg} / \mathrm{dL}$. Furthermore, the proposed sensor can be reused 10 times at an acceptable performance level. Although multiple nanowires sensors have been demonstrated and literatures [3,4] have pointed out the advantages and drawbacks of the nanowire sensor for measuring glucose concentration, to the best of our knowledge, their reusability and resolution at various nanowire lengths have not been reported.

\section{Fabrication and Characteristic of the Proposed Sensor}

\subsection{Fabrication Procedure of the Proposed Sensor}

The proposed nanowire sensor was fabricated using a top-down method [3,10], and the Si nanowire was nanopatterning through I-line lithography. A $35 \mathrm{~nm}$ thick oxide layer was grown at $900{ }^{\circ} \mathrm{C}$ through thermal oxidation, following which a $30 \mathrm{~nm}$ thick $\mathrm{Si}_{3} \mathrm{~N}_{4}$ was deposited at $780{ }^{\circ} \mathrm{C}$ through low pressure chemical vapor deposition method (LPCVD). Next, an approximately $2 \mathrm{~nm}$ thick layer was grown on the Si substrate through thermal oxidation at $900{ }^{\circ} \mathrm{C}$ and used as the bottom dielectric layer. A $60 \mathrm{~nm}$ thick poly-Si layer was deposited for the channel through LPCVD at $550{ }^{\circ} \mathrm{C}$. Subsequently, I-line lithography was performed along with the photoresistor trimming process, followed by Si etching. Therefore, a slender channel and a source/drain pad region were fabricated on the bottom dielectric layer. Next, the dimensions of poly-Si nanowires were trimmed through a re-oxidation procedure for forming poly-Si nanowires with a width of approximately $340 \mathrm{~nm}$. Figure 1a schematically depicts the device structure and measurement setup and Figure $1 \mathrm{~b}$ shows the scanning electron microscope (SEM) image of the top-view of the device. In this study, the sensor contained nanowires of three lengths fabricated on the same chip for comparing their electrical characteristics under the same manufacturing conditions. 
After fabricating the multiple nanowires sensor, the surface of nanowire was modified by treating with 10\% (v/v) 3-aminopropyltriethoxysilane (APTES) in ethanol for $15 \mathrm{~min}$ at room temperature, followed by heating at $100{ }^{\circ} \mathrm{C}$ for $45 \mathrm{~min}$. The surface were covered with $0.01 \%$ suberic acid bis(3-sulfo-N-hydroxysuccinimide ester) sodium salt (BS3) in a $10 \mathrm{mM}$ phosphate buffered saline (PBS) solution for $30 \mathrm{~min}$ at room temperature. This procedure was used to modify the nanowire surface on which $\mathrm{GO}_{x}$ was immobilizing on $\mathrm{SiO}_{2}$ through covalent bonding. Then the modified surface was covered with a $\mathrm{GO}_{x}$ solution having a $\mathrm{pH}$ value of 7 and a concentration of $150 \mu \mathrm{g} / \mathrm{mL}$ for $1 \mathrm{~h}$. Unreacted aldehyde groups were quenched by immersing in a $15 \mathrm{mM}$ Tris buffer solution for $15 \mathrm{~min}$ at room temperature [11].

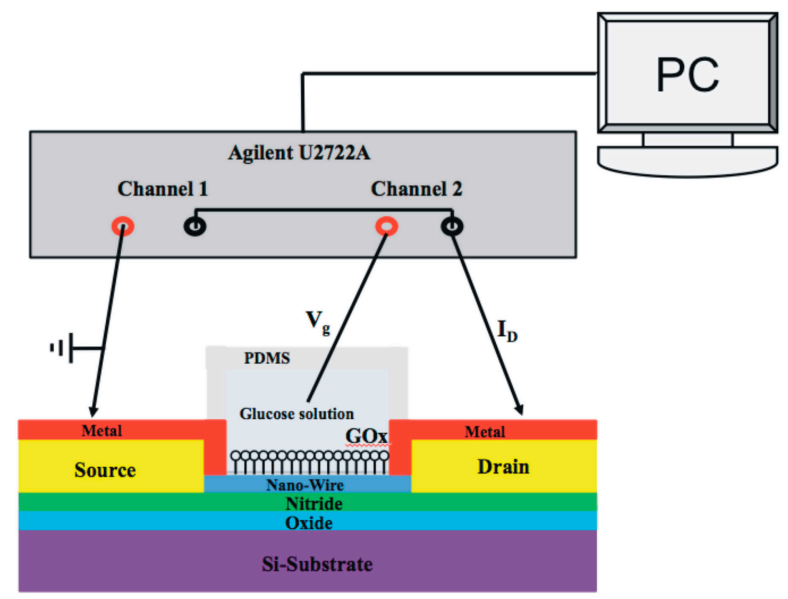

(a)

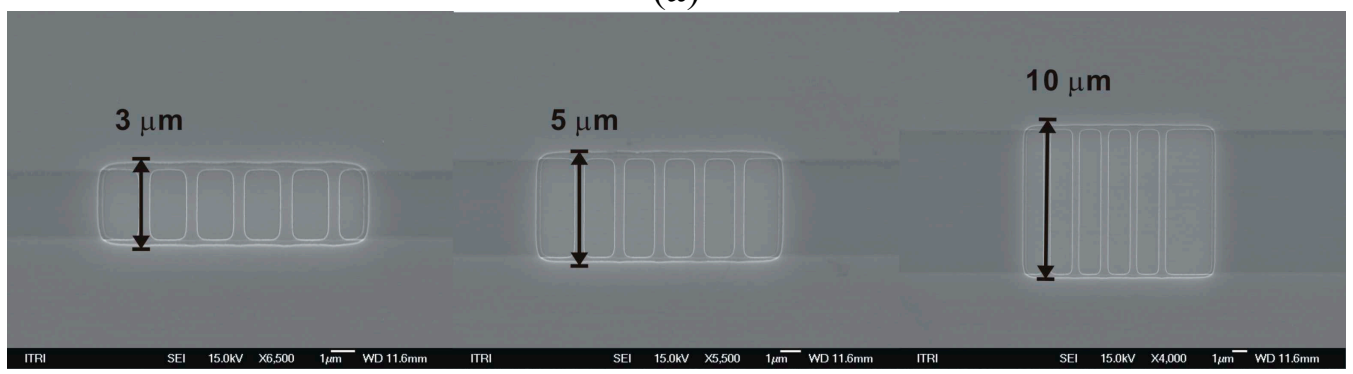

(b)

Figure 1. (a) Diagram of the device structure and the experimental setup; (b) Top-view scanning electron microscope (SEM) image of the proposed sensor containing various nanowire lengths.

\subsection{Characteristic of the Proposed Sensor}

The electrical behavior of nanowire can be described by the $I_{\mathrm{D}}-V_{\mathrm{D}}$ and $I_{\mathrm{D}}-V_{\mathrm{G}}$ curves. Varying the width, length, and number of the nanowires in the sensor can alter the $I_{\mathrm{D}}-V_{\mathrm{D}}$ and $I_{\mathrm{D}}-V_{\mathrm{G}}$ behaviors. Therefore, an appropriate arrangement of the device with a high sensitivity and low threshold voltage can be designed for biosensing. Eflstrom et al. [9] predicted that the nanowire width 100-150 nm can preserve the threshold voltage within $2.2-1.5 \mathrm{~V}$. Their results further indicated that the device sensitivity increased as the width of nanowire decreased. Hsu et al. [10] demonstrated the sensitivity of various nanowire lengths and determined that shorter nanowire length led to higher sensitivity. In this study, we fabricated a glucose sensor with five parallel nanowires with various nanowire lengths 
$(3,5$, and $10 \mu \mathrm{m})$. The electrical properties of the proposed sensor were evaluated before measuring the glucose concentration. The $I_{\mathrm{D}}-V_{\mathrm{D}}$ curve, showed in Figure $2 \mathrm{a}$, indicates that the drain current of the proposed sensor saturates if the drain voltage exceeds $1 \mathrm{~V}$. Therefore, the drain voltage was maintained at $1 \mathrm{~V}$ to preserve sensor operation in the linear response region. Figure $2 \mathrm{~b}$ demonstrates the threshold voltage $V_{\text {th }}$ of the proposed sensor, which is determined as the intercept on the $V_{\mathrm{G}}$-axis in the $I_{\mathrm{D}}-V_{\mathrm{G}}$ curve [8]. The results showed that $V_{\text {th }}$ decreases as the nanowire length increase.

(a)

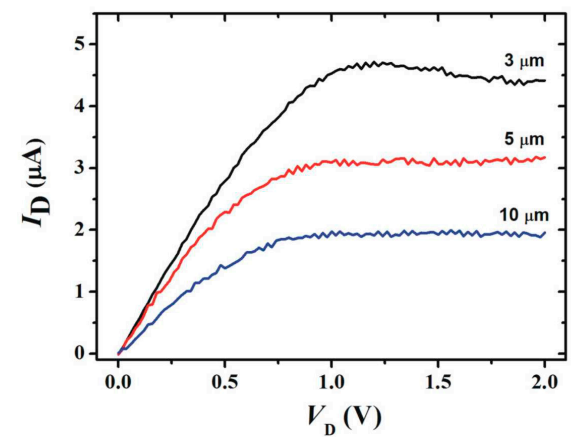

(b)

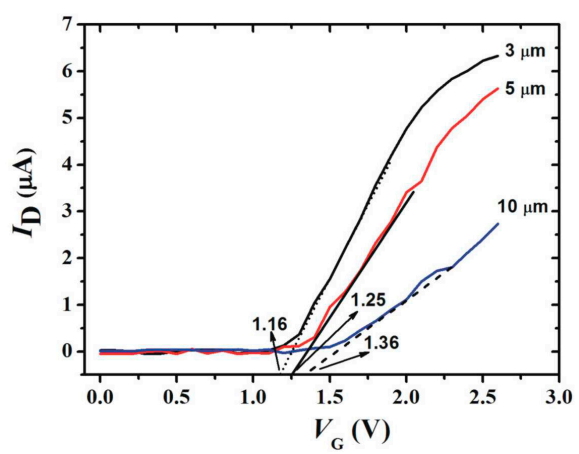

Figure 2. (a) $I_{\mathrm{D}}-V_{\mathrm{D}}$ measurement for different fabricated poly-Si nanowires; (b) $I_{\mathrm{D}}-V_{\mathrm{G}}$ measurement for different fabricated poly-Si nanowires.

\section{Results and Discussion}

Performance of the proposed nanowire sensor was investigated in glucose solution of concentrations varying from 10 to $300 \mathrm{mg} / \mathrm{dL}$. The environment temperature controlled at $25^{\circ} \mathrm{C}$ for each measurement. When the solution was dripped onto the sensor, electron distribution around the nanowire varied as the glucose reacted with the $\mathrm{GO}_{x}$ and converted into gluconic acid and hydrogen peroxide. The chemical reaction is formulated as follows:

$$
\text { Glucose }+\mathrm{O}_{2} \stackrel{\mathrm{GO} x}{\longrightarrow} \text { Gluconic acid }+\mathrm{H}_{2} \mathrm{O}_{2}
$$

Figure 3 shows the current-time response curve of the multiple nanowire sensor during the successive additions of glucose solution. Figure 3 shows the increase in drain current with the glucose concentration. At glucose concentrations lower than $100 \mathrm{mg} / \mathrm{dL}$, a $3 \mu \mathrm{m}$ nanowire sensor can easily distinguish the drain current variation. By contrast, the drain current variation measured using the sensor with $10 \mu \mathrm{m}$ nanowire changed to approximately $0.1 \mu \mathrm{A}$. Furthermore, the total drain current variation measured using the sensors with the 5 and $10 \mu \mathrm{m}$ nanowires was approximately $0.5 \mu \mathrm{A}$, in which one half of variation of the results was measured using a sensor with the $3 \mu \mathrm{m}$ nanowire.

Reusability of the proposed sensor was then verified through ten successive measurements of a glucose solution concentration of $300 \mathrm{mg} / \mathrm{dL}$ (Figure 4a). Obviously, the drain current variation decreased as the proposed sensor exceeded 10 applications because of the gluconic acid (reacting product) being absorbed by the nanowire and the increased resistance of the sensor. Figure $4 \mathrm{~b}$ depicts the SEM image of the top-view of the sensor, which underwent 20 applications. The gluconic acid was absorbed by the device and was deposited at the nanowire around the regions of source and drain. Therefore, the proposed sensor can be used up to 10 times at similar functionality. The enlarged diagram 
of lower- $I_{\mathrm{d}}$ region also inset in Figure $4 \mathrm{a}$ and indicated that good behavior of return-to-zero. To prevent the absorbtion of the gluconic acid, the direction of flow channels can be aligned with the nanowire direction. The injection system could be replaced by an automatic injector which provided continuous injection of DI water for cleaning the device.

(a)

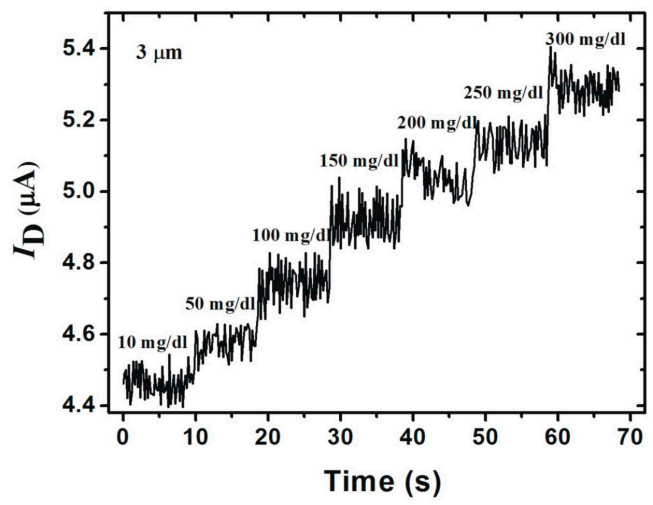

(b)

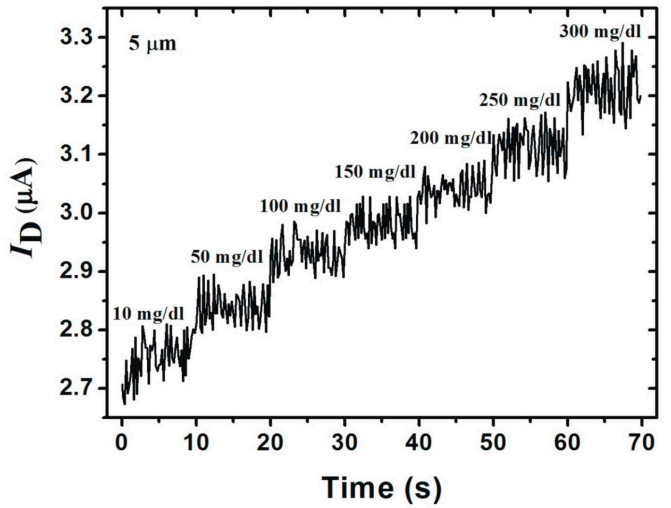

(c)

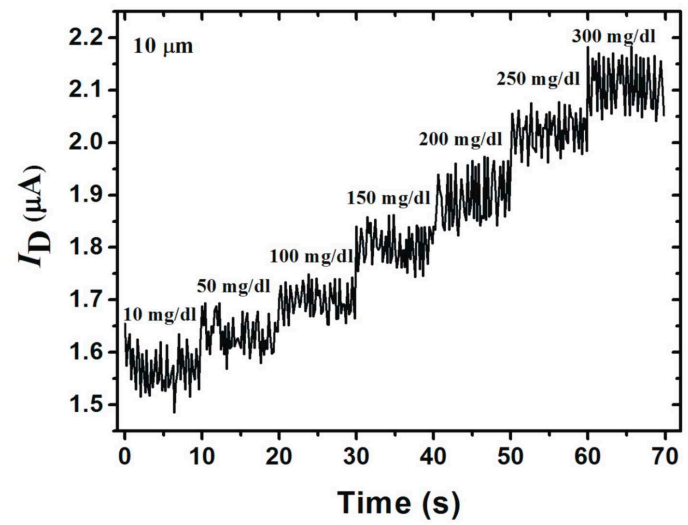

Figure 3. Current-time response curve of the proposed sensor with nanowire lengths of (a) $3 \mu \mathrm{m}$, (b) $5 \mu \mathrm{m}$ and (c) $10 \mu \mathrm{m}$.

(a)

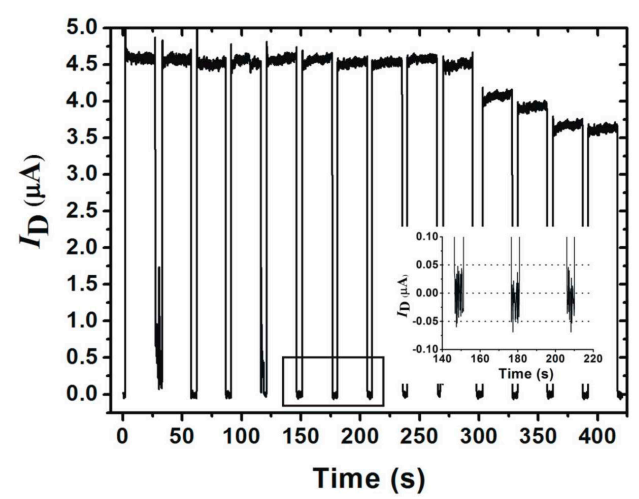

(b)

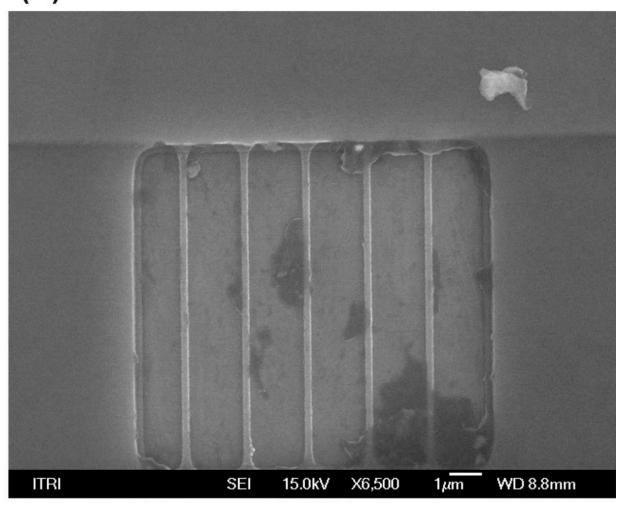

Figure 4. (a) Reusability of the proposed sensor; (b) Top-view SEM image of the proposed sensor used more than 10 applications. 
Figure 5 shows the resulting calibration curve measured by the proposed sensors; $\square, \bigcirc, \Delta$, and I represent the average value of 10 measured data sets and the standard deviation of each concentration measured by the proposed sensor with various nanowire lengths. Resolutions of these sensors can be represented as:

$$
c=\frac{I}{s}
$$

where $s$ is the slope of the calibration curve; $\Delta I$ and $\Delta c$ represent the resolution of the measured current and concentration, respectively. The slope of the calibration curve indicates the sensitivity of the proposed sensor; clearly, sensitivity increases with decreasing nanowire length. The shorter nanowire length leads to smaller device dimensions and resistance becomes higher. The surface-area-to-volume ratio will increase with smaller dimensions of the nanowire device which results in greater surface effects on the electrical conduction. Therefore, the sensitivity increases with shorter nanowire length $[12,13]$. This finding correlates with the $I_{\mathrm{D}}-V_{\mathrm{G}}$ curves in Figure $2 \mathrm{~b}$. Shaper slope indicates higher sensitivity.

Furthermore, the measured current resolution (depending on the current meter) affects the resolution of the concentration. Considering the resolution of current meter, which indicated the theoretical resolution, the resolution of measured current was $0.1 \mathrm{nA}$. However, the solution temperature, electrode conductivity, and unexpected electrical noise of the measurement apparatus influence the current. Therefore, the actual resolution of the measured current is obtained by the current fluctuation as constant $V_{\mathrm{G}}$ is applied to the DI water sample. In this work, the current fluctuation is approximately $37 \mathrm{nA}$ within $10 \mathrm{~s}$ (Figure 6).

(a)

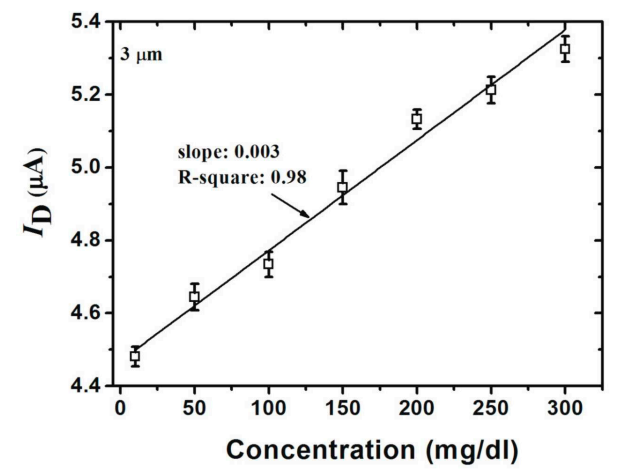

(b)

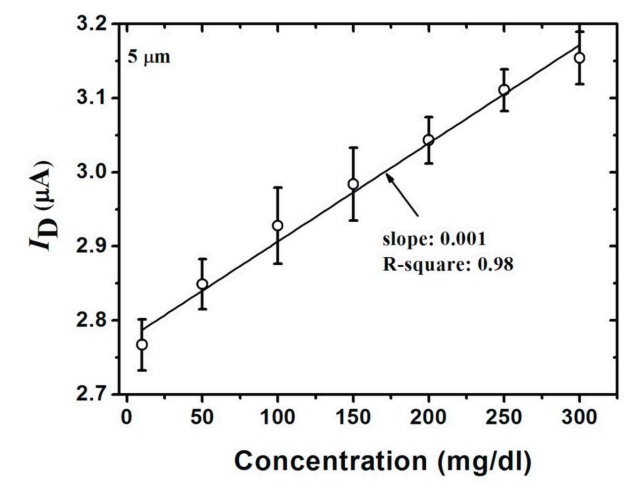

(c)

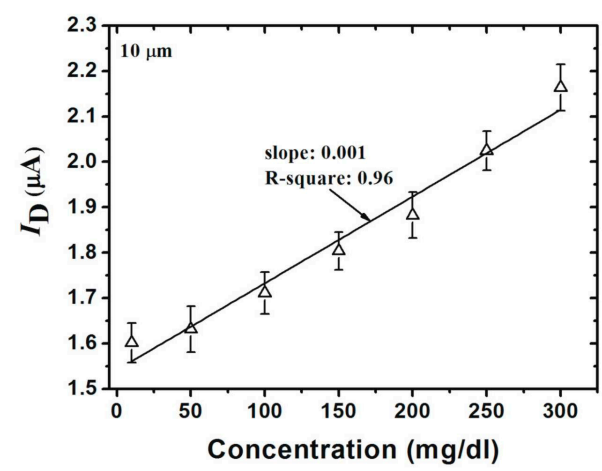

Figure 5. Calibration curves of the proposed sensor with nanowire lengths of (a) $3 \mu \mathrm{m}$, (b) $5 \mu \mathrm{m}$ and (c) $10 \mu \mathrm{m}$. 


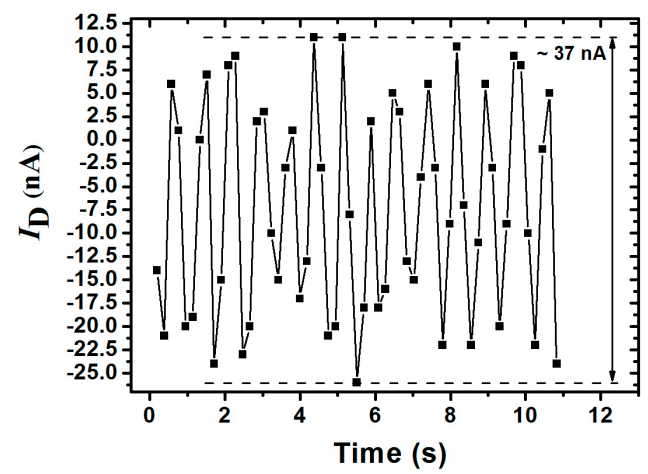

Figure 6. Current fluctuation in the proposed sensor.

The resolution of these sensors was calculated according to Equation (2) and summarized in Table 1. Obviously, resolution value of the proposed sensor decreases with an increasing nanowire length. The optimal resolutions of the proposed sensor were approximately $0.003 \mathrm{mg} / \mathrm{dL}$ (theoretical) and $1.23 \mathrm{mg} / \mathrm{dL}$ (practical) respectively, which was achieved by using the proposed sensor with a nanowire of $3 \mu \mathrm{m}$ in length.

Table 1. Sensitivity and resolution of proposed sensors with various nanowire lengths.

\begin{tabular}{ccccc}
\hline \multirow{2}{*}{$\begin{array}{c}\text { Length } \\
(\boldsymbol{\mu} \mathbf{m})\end{array}$} & $\boldsymbol{V}_{\text {th }}(\mathbf{V})$ & $\begin{array}{c}\text { Sensitivity }(\boldsymbol{s}) \\
(\boldsymbol{\mu} \mathbf{A} /(\mathbf{m g} / \mathbf{d L}))\end{array}$ & Theoretical $(\Delta \boldsymbol{I}=\mathbf{0 . 1} \mathbf{~ n A})$ & Real $(\Delta \boldsymbol{I}=\mathbf{3 7} \mathbf{n A})$ \\
\hline 3 & 1.16 & 0.03 & 0.003 & 1.23 \\
5 & 1.25 & 0.01 & 0.01 & 3.7 \\
10 & 1.36 & 0.01 & 0.01 & 3.7 \\
\hline
\end{tabular}

\section{Conclusions}

This study demonstrates the feasibility of a non-doping enzymatic multiple nanowires sensor for measuring the glucose concentration. Experimental results indicated that the threshold voltage of the sensor increases with an increasing the length of nanowire. The smallest threshold voltage was approximately $0.88 \mathrm{~V}$. In addition, the resolution of the proposed nanowire sensor was directly proportional to the length of the nanowire. Moreover, the theoretical resolution was approximately $0.003 \mathrm{mg} / \mathrm{dL}(0.17 \mu \mathrm{mol} / \mathrm{L})$ and the smallest concentration variation can be determined was $10 \mathrm{mg} / \mathrm{dL}(0.56 \mathrm{mmol} / \mathrm{L})$. This study further demonstrated that reusing the proposed sensor for 10 applications sustains an acceptable sensitivity and resolution.

\section{Acknowledgments}

The authors would like to thank the National Science Council of the Republic of China for financially supporting this research under Contract No. NSC 102-2221-E-076-MY3.

\section{Author Contributions}

Cheng-Chih Hsu designed the multiple-nanowire glucose sensors and authored the manuscript. Yu-Ching Liao fabricated the sensors and evaluated their electrical characterization. Hsin-I Yeh and Yen-Ting Tsai measured and analyzed the data. Chyan-Chyi Wu designed the readout circuit for the sensors. All authors have read and approved the final manuscript. 


\section{Conflicts of Interest}

The authors declare no conflict of interest.

\section{References}

1. National Diabetes Statistics Report, 2014 Edition. Available online: http:www.cdc.gov/diabetes/ pubs/statsreport14/national-diabetes-report-web.pdf (accessed on 3 July 2015).

2. IDF Diabetes Atlas, 6th Edition. Available online: http://www.idf.org/sites/default/files/ EN_6E_Atlas_Full_0.pdf (accessed on 13 August 2015).

3. Chen, K.I.; Li, B.R.; Chen, Y.T. Silicon nanowire field-effect transistor-based biosensors for biomedical diagnosis and cellular recording investigation. Nano Today 2011, 6, 131-154. [CrossRef]

4. Rahman, M.M.; Ahammad, A.J.S.; Jin, J.H.; Ahn, S.J.; Lee, J.J. A comprehensive review of glucose biosensors based on nanostructured metal-oxides. Sensors 2010, 10, 4855-4886. [CrossRef] [PubMed]

5. Yang, C.L.; Zhang, X.H.; Lan, G.; Chen, L.Y.; Chen, M.W.; Zeng, Y.Q.; Jiang, J.Q. Pd-based nanoporous metals for enzyme-free electrochemical glucose sensors. Chin. Chem. Lett. 2014, 25, 496-500. [CrossRef]

6. Su, S.; Wei, X.; Guo, Y.; Zhong, Y.; Su, Y.; Huang, Q.; Fan, C.; He, Y. A silicon nanowire-based electrochemical sensor with high sensitivity and electrocatalytic activity. Part. Part. Syst. Charact. 2013, 30, 326-331. [CrossRef]

7. Zang, J.; Li, C.M.; Cui, X.; Wang, J.; Sun, X.; Dong, H.; Sum, C.Q. Tailoring zinc oxide nanowires for high performance amperometric glucose sensor. Electroanalysis 2007, 19, 1008-1014. [CrossRef]

8. Sze, S.M.; Lee, M.K. Semiconductor Devices Physics and Technology, 3rd ed.; John Wiley \& Sons: Hoboken, NJ, USA, 2013.

9. Elfstrom, N.; Juhasz, R.; Sychugov, I.; Engfeldt, T.; Karlstrom, A.E.; Linnros, J. Surface charge sensitivity of silicon nanowires: size dependence. Nano. Lett. 2007, 7, 2608. [CrossRef] [PubMed]

10. Hsu, C.C.; Yang, C.Y.; Lai, C.J.; Dai, C.L. Optimization of reusable polysilicon nanowire sensor for salt concentration measurement. Jpn. J. Appl. Phys. 2014, 53, 06JE04. [CrossRef]

11. Lin, T.Q.; Lu, Y.L.; Hsu, C.C. Fabrication of glucose fiber sensor based on immobilized GOD technique for rapid measurement. Opt. Express 2010, 18, 27560-27566. [CrossRef] [PubMed]

12. Rahman, S.F.A.; Yusof, N.A.; Hashim, U.; Nuzaihan Md Nor, M. Design and fabrication of silicon nanowire based sensor. Int. J. Electrochem. Sci. 2013, 8, 10946-10960.

13. Park, I.; Li, Z.; Pisano, A.P.; Williams, R.S. Towards the silicon nanowire-based sensor for intracellular biochemical detection. Biosens. Bioelectron. 2007, 22, 2065-2070. [CrossRef] [PubMed]

(C) 2015 by the authors; licensee MDPI, Basel, Switzerland. This article is an open access article distributed under the terms and conditions of the Creative Commons Attribution license (http://creativecommons.org/licenses/by/4.0/). 